\title{
Effect of microstructure anisotropy on the deformation of MAX polycrystals studied by in-situ compression combined with neutron diffraction
}

\author{
A. Guitton, ${ }^{1}$ S. Van Petegem, ${ }^{2}$ C. Tromas, ${ }^{2}$ A. Joulain, ${ }^{1}$ H. Van Swygenhoven, ${ }^{2}$ \\ and L. Thilly ${ }^{1, a)}$ \\ ${ }^{1}$ Institut Pprime, CNRS-University of Poitiers-ENSMA, SP2MI, 86962 Futuroscope, France \\ ${ }^{2}$ Materials Science and Simulations, NUM/ASQ, CH5232 Villigen PSI, Switzerland
}

(Received 8 April 2014; accepted 10 June 2014; published online 19 June 2014)

\begin{abstract}
In situ compression tests combined with neutron diffraction were performed on $\mathrm{Ti}_{2} \mathrm{AlN}$ MAX polycrystals with lamellar anisotropic microstructure: the diffraction peak evolution (position and profile) with applied stress reveals that lamellar grains parallel to compression axis remain elastic while lamellar grains perpendicular to compression plastify, both families being subjected to strong variations of heterogeneous strains (types II and III). We demonstrate that this behavior originates from the complex response of the very anisotropic lamellar microstructure and explains the observation of reversible hysteretic loops when cycling MAX polycrystals even in the elastic regime. (C) 2014 AIP Publishing LLC. [http://dx.doi.org/10.1063/1.4884601]
\end{abstract}

MAX phases are nanolaminated ternary carbides or nitrides with hexagonal lattice. ${ }^{1}$ They show a unique combination of metal-like and ceramic-like properties such as high electrical conductivity, machinability, high stiffness, temperature, and corrosion resistance. ${ }^{1}$ Regarding the mechanical properties, they exhibit a brittle-to-ductile transition around $800^{\circ} \mathrm{C}$ which leads to massive failure after a very limited plastic regime at room temperature (RT) ${ }^{1,2}$ A very intriguing behaviour is observed when MAX samples are subjected to load-unload cycles in the elastic regime: reversible hysteretic loops are recorded. ${ }^{3}$ These features are, respectively, illustrated in this study by the compression stress-strain curves shown in Fig. 1 obtained on $\mathrm{Ti}_{2} \mathrm{AlN}$ MAX polycrystal. The formation of open loops suggests an energy dissipative process although in the elastic regime: a "kinking non-linear elastic" model has been developed involving Incipient Kink Bands (IKBs), that are entities composed of dislocation loops lying in parallel basal planes and stacked on top of each other. ${ }^{4,5}$ The IKBs are supposed to nucleate and expand when load is applied and to collapse when load is removed. This "reversible" dislocation-based micro-mechanism is invoked to explain the above-mentioned energy dissipation observed in the elastic regime. ${ }^{3-5}$ Besides the fact that IKBs have never been observed, the observation of open-loops at very low stress somehow contradicts this model involving a critical shear stress needed to overcome the energy barrier associated to dislocation nucleation. In a very recent work, Jones et al. ${ }^{6}$ have shown, by in-situ deformation under $\mathrm{x}$-rays and simulation, that the MAX polycrystal can be described as an ensemble of soft and hard grains, with each grain having a limited number of available slip systems due to the hexagonal lattice. The reversible hysteretic behaviour can therefore be explained by this plastic anisotropy and the associated Bauschinger effect, i.e., within the classical framework of crystal plasticity in polycrystals.

An aspect which was however not taken into account in the mechanical behaviour so far is that the MAX polycrystals,

\footnotetext{
${ }^{\text {a) }}$ Author to whom correspondence should be addressed. Electronic mail: ludovic.thilly@univ-poitiers.fr
}

fabricated by powder metallurgy techniques, exhibit very anisotropic microstructure with grains being highly elongated in the basal plane direction, as illustrated in Fig. 2(a) for $\mathrm{Ti}_{2} \mathrm{AlN}$. Such complex grain morphology and associated grain interactions are expected to play a major role in the mechanical response of MAX polycrystals, as already suggested in $\mathrm{Ti}_{2} \mathrm{AlC}$ and $\mathrm{Ti}_{3} \mathrm{SiC}_{2} \cdot{ }^{6,7}$

In this Letter, we combine compression tests, neutron diffraction, and Scanning Electron Microscopy (SEM) observations to uncover further aspects of the deformation of MAX polycrystals by taking into account the real microstructure features of these complex materials.

Fully dense $\mathrm{Ti}_{2} \mathrm{AlN}$ samples were prepared by powder metallurgy based on hot isostatic pressing: ${ }^{8}$ all the details of the synthesis are given in Ref. 8. Two types of microstructures were produced: (1) mean grain size of $17 \mu \mathrm{m}$ (standard deviation: $9 \mu \mathrm{m}$ ), labelled "small-grain;" (2) bimodal distribution around $20 \mu \mathrm{m}$ and $88 \mu \mathrm{m}$ (standard deviation: $12 \mu \mathrm{m}$ ), labelled "coarse-grain." Both microstructures exhibit the previously described anisotropic grain morphology (Fig. 2(a)). In the following, only the results recorded for "coarse-grain" samples are given but the same conclusions can be drawn for "small-grain" samples, the only difference being a higher elastic limit observed for the latter as a result of gain refinement strengthening ( $\sim 500 \mathrm{MPa}$ vs. $\sim 300 \mathrm{MPa}$ ).

The experiments were conducted at the Swiss spallation neutron source (SINQ, Paul Scherrer Institut, Switzerland) using the high resolution time-of-flight diffractometer with multiple pulse overlap (POLDI), with a polychromatic beam and a beam size of $4 \times 4 \mathrm{~mm}^{2}$. . The neutron beam is oriented at $45^{\circ}$ from the compression axis (CA), the one-dimensional position-sensitive ${ }^{3} \mathrm{He}$-detector being positioned at $90^{\circ}$ from the incident beam, with an angular range of $\pm 15^{\circ}$. Two types of in situ compression tests were performed at room temperature on samples with dimensions of $4 \times 4 \times 10 \mathrm{~mm}^{3}$, at a strain rate of $1.2 \times 10^{-4} \mathrm{~s}^{-1}$ : monotonous or cyclic compressions, as seen in Fig. 1. In the first case, the compression was paused at intermediate stress states to record the neutron diffractograms (during more than $4 \mathrm{~h}$, after $15 \mathrm{~min}$ relaxation) up to the (partial) failure of the samples (Fig. 1(a)); in the 


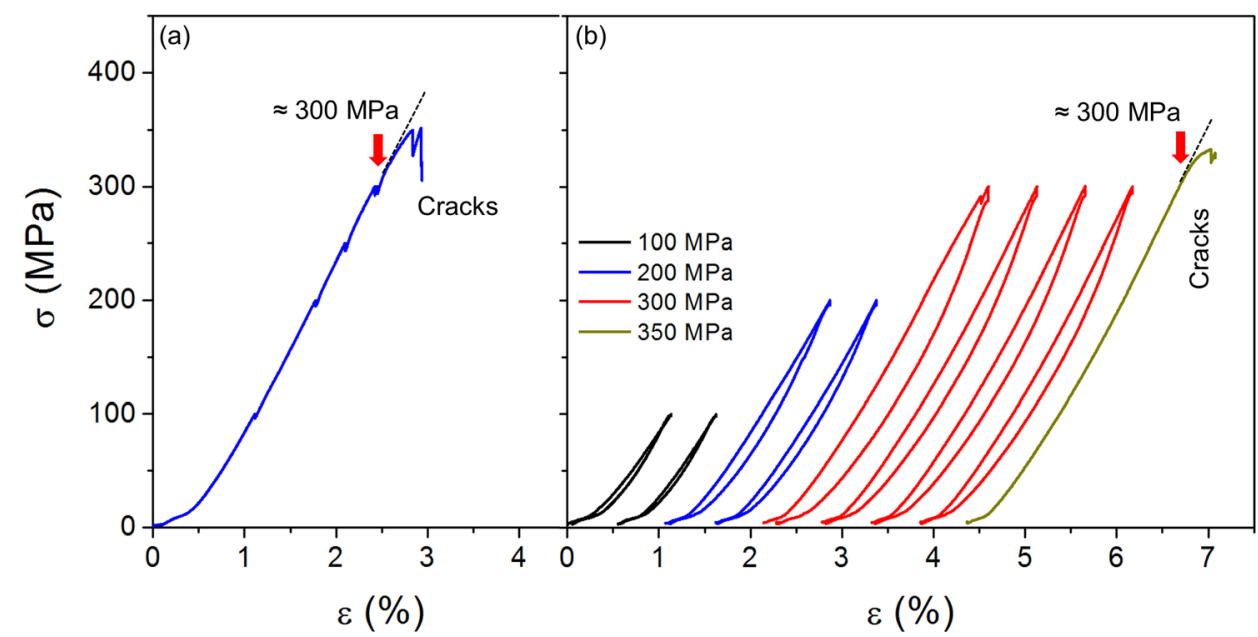

FIG. 1. RT stress-strain compression curves obtained in-situ at the POLDI beamline on coarse-grain $\mathrm{Ti}_{2} \mathrm{AlN}$ MAX polycrystals: (a) during monotonous compression and (b) during loadunload cycles (each cycle is artificially shifted for clarity). second case, diffracted neutrons are collected at unloaded and loaded states ( 2 cycles at $100 \mathrm{MPa}$ and $200 \mathrm{MPa}, 4$ cycles at $300 \mathrm{MPa}$ ) (Fig. 1(b)). In all cases, neutrons are scattered on $\{$ hkil $\}$ planes that are perpendicular to the CA: each (hkil) peak of the collected diffractogram is associated to an ensemble of grains (a grain family) having in common $\{$ hkil $\}$ planes perpendicular to $\mathrm{CA}$; the angle between (hkil) planes and basal (0001) planes being known, it is possible to assign each (hkil) peak a value of the angle $\alpha$ between the normal to basal plane (NBP) and CA. Since most grains are elongated in the basal plane direction, each (hkil) peak is associated to a set of lamellar grains having the same orientation vs. CA: for example, the (0001) peak corresponds to $\alpha=0^{\circ}$, i.e., lamellar grains perpendicular to CA (see Fig. 2(b)).

After indexation of the diffractograms, the highest intensity peaks are individually fitted by single Gaussian functions that provide, among other parameters, the peak position, $\mathrm{d}_{\text {hkil }}$, and the peak full width at half maximum, $\mathrm{W}_{\text {hkil }}$, with respective errors of $5 \times 10^{-5} \AA$ and $2 \times 10^{-4} \AA$ (in the following, error bars are always smaller than symbol size). The lattice (elastic) strain is then calculated as $\varepsilon_{\text {el }}=\Delta \mathrm{d} / \mathrm{d}^{0}=\left(\mathrm{d}_{\text {hkil }}-\mathrm{d}_{\text {hkil }}{ }^{0}\right) / \mathrm{d}_{\text {hkil }}{ }^{0}$, where $\mathrm{d}_{\text {hkil }}{ }^{0}$ is the peak position in unloaded initial state. The relative peak width is calculated as $\Delta \mathrm{w} / \mathrm{w}^{0}=\left(\mathrm{w}_{\text {hkil }}-\mathrm{w}_{\text {hkil }}{ }^{0}\right) / \mathrm{w}_{\text {hkil }}{ }^{0}$, where $\mathrm{w}_{\text {hkil }}{ }^{0}$ is the peak width in unloaded initial state.

During compression, the lattice strain $\varepsilon_{\mathrm{el}}$ is expected to become negative. The decrease of $\varepsilon_{\mathrm{el}}$ is first linear as a function of applied stress $\sigma$ (elastic regime) whatever the grain
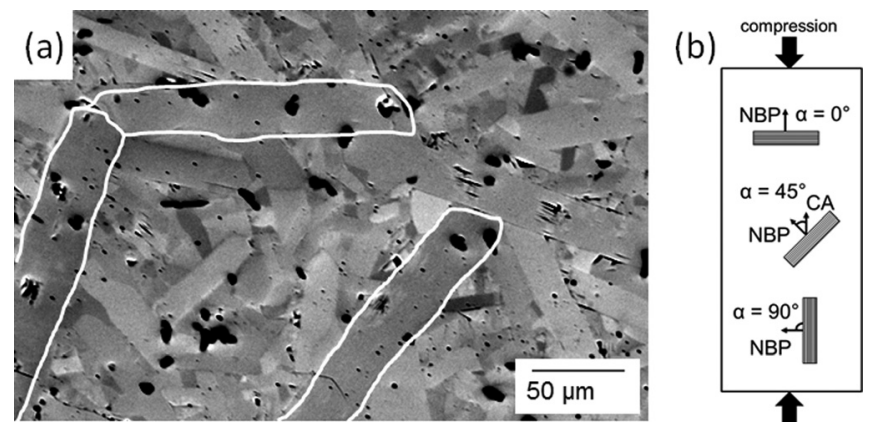

FIG. 2. SEM micrograph of coarse-grain $\mathrm{Ti}_{2} \mathrm{AlN}$ MAX polycrystal, with emphasis on the lamellar shape of the microstructure. (b) Definition of the angle $\alpha$ between the NBP and the CA in different grain families. family; then depending on the orientation of the grains, some experience plasticity leading to a stagnation of $\varepsilon_{\mathrm{el}}$ (a decrease of the slope $\left|\partial\left(\varepsilon_{\mathrm{e} 1}\right) / \partial(\sigma)\right|$ ), while other elastic grains experience an increase of $\varepsilon_{\mathrm{el}}$ (an increase of $\left.\left|\partial\left(\varepsilon_{\mathrm{el}}\right) / \partial(\sigma)\right|\right)$. Such behaviour is due to load transfer from plastic to elastic grains. ${ }^{10,11}$ Regarding peak width, besides grain size that is considered constant during the compression tests, the evolution of $w_{\text {hkil }}$ is a footprint of heterogeneous strains in the grain family, which origin is type II (grain to grain stress variation because of polycrystal effect) and type III (dislocations) microstresses. ${ }^{11}$

Fig. 3(a) presents the evolution of the lattice strain at different applied stresses for 6 \{hkil\} grain families, each associated to a different $\alpha$ value, ranging from $27.8^{\circ}$ to $81.8^{\circ}$ (i.e., corresponding to lamellar grains being more and more parallel to CA). Below 200-250 MPa, the lattice strain of each grain family behaves linearly as a sign of elastic deformation ("domain 1-elastic"). This result can be directly compared to the macroscopic information obtained from monotonous and cyclic applied stress-strain curves (Fig. 1) showing that the macroscopic elastic limit is reached around $300 \mathrm{MPa}$. Above this threshold, two opposite behaviours are

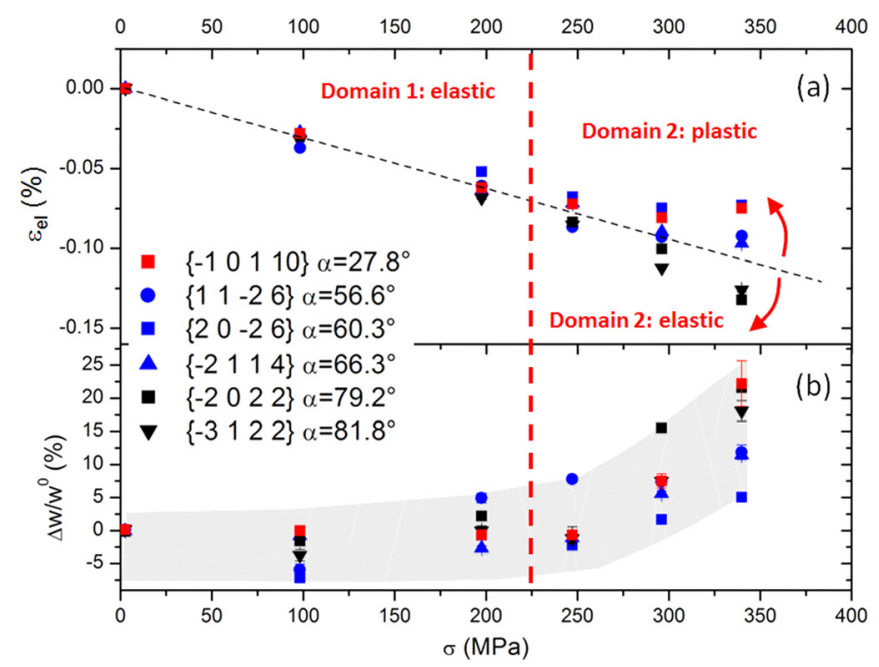

FIG. 3. (a) Evolution of the lattice strain $\left(\varepsilon_{\mathrm{el}}\right.$ in $\left.\%\right)$ versus applied stress measured from different $\{$ hkil $\}$ reflections in grain families where the orientation of the basal plane is given by the angle $\alpha$ defined in Fig. 2(b). (b) Relative evolution of the peak width $\left(\Delta \mathrm{w} / \mathrm{w}^{0}\right.$ in $\left.\%\right)$ versus applied stress for the same $\{$ hkil $\}$ reflections (shaded area is a guide to the eye). 


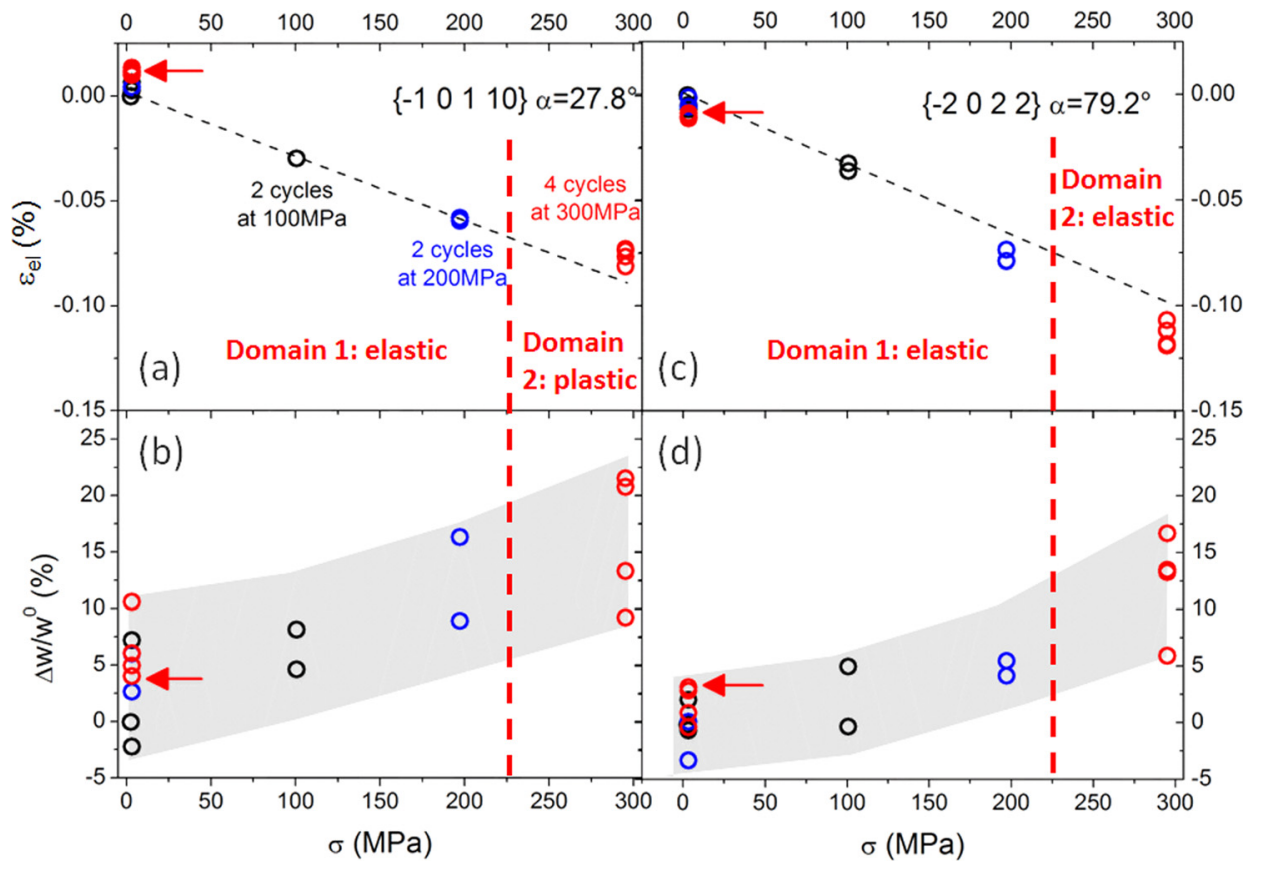

FIG. 4. (a) Lattice strain versus applied stress measured from $\left\{\begin{array}{llll}-1 & 0 & 1 & 10\end{array}\right\}$ reflection during cyclic compression test (neutrons being collected sequentially at unloaded and loaded states); the arrow indicates the final unloaded state. (b) Evolution of the relative peak width versus applied stress measured from $\left\{\begin{array}{llll}-1 & 0 & 1 & 10\end{array}\right\}$ reflection. (c) and (d) Similar plots for the $\left\{\begin{array}{llll}-2 & 0 & 2 & 2\end{array}\right\}$ reflection. Shaded areas are guides to the eye and correspond to the uncertainty of fit repeatability. observed in the diffraction data: the slope $\left(\left|\partial\left(\varepsilon_{\mathrm{el}}\right) / \partial(\sigma)\right|\right)$ of lattice strain starts to decrease in grain families with $\alpha<70^{\circ}$ while the slope increases in grain families with $\alpha>70^{\circ}$. We can therefore consider "domain 2-plastic" and "domain 2-elastic" to be, respectively, associated to "plastic grains" and "elastic grains," with here an obvious threshold between the plastic/elastic behaviour, at $\alpha \approx 70^{\circ}$. On Fig. 3(b), the evolution of the relative peak width with applied stress is shown: surprisingly, there is no dependence of $\Delta \mathrm{w} / \mathrm{w}^{0}$ with respect to $\alpha$, i.e., all grain families exhibit a noticeable increase of heterogeneous strains in domain 2. In the "plastic grains" $\left(\alpha<70^{\circ}\right)$, this increase is related to the presence of dislocations, i.e., type III microstrains, which cannot be the case in "elastic grains."

Fig. 4 brings further credit to these observations by showing the same quantities (lattice strain and relative peak width) as a function of applied stress during the cyclic compression. Two extreme cases are considered: (1) the $\left\{\begin{array}{llll}-1 & 0 & 1 & 10\end{array}\right\}$ grain family with $\alpha=27.8^{\circ}$ (lamellar grains close to perpendicular to CA) in Figures 4(a) and 4(b); (2) the $\left\{\begin{array}{llll}-2 & 0 & 2 & 2\end{array}\right\}$ grain family with $\alpha=79.2^{\circ}$ (lamellar grains close to parallel to CA) in Figures 4(c) and 4(d). The first grain family obviously reaches the plastic regime (decrease of $\left.\left|\partial\left(\varepsilon_{\mathrm{el}}\right) / \partial(\sigma)\right|\right)$ when cycled at $300 \mathrm{MPa}$, as confirmed by the build-up of a small residual tensile strain after final unloading (arrow on Fig. 4(a)). The second grain family remains in the elastic regime (increase of $\left|\partial\left(\varepsilon_{\mathrm{el}}\right) / \partial(\sigma)\right|$ ), even at $300 \mathrm{MPa}$, and undergoes a load transfer from plastic grains that is retained in the form of a small residual compressive strain after final unloading (arrow on Fig. 4(c)). In both cases, the relative peak width increases in a reversible manner (within experimental and fit uncertainties) with loadunload cycles, suggesting that the microstresses evolve in both grain families: only type II in elastic families (large $\alpha$ value), type II and type III in plastic families (small $\alpha$ value). And this, as the macroscopic stress-strain curves exhibit widely open reversible loops at $300 \mathrm{MPa}$ (Fig. 1(b)).
Fig. 5(a) presents a post-mortem SEM observation of the sample surface showing that the "coarse-grain" polycrystal can be seen, as a first approximation, as composed of coarse lamellar grains connected to each other and embedded in a polycrystalline "matrix" (as schematized in Fig. 5(b)). Interestingly, the coarse grains can be divided into two types: (1) the lamellar grains close to the perpendicular to CA (i.e., with low $\alpha$ value) exhibit slip traces (and cracks but we shall not discuss this aspect here) as a clear footprint of dislocation activity and (2) the lamellar grains close to the parallel to CA (i.e., with high $\alpha$ value) show no sign of plastic events suggesting a purely elastic behaviour. These surface observations (similar to those observed in the "small grain" samples) have been similarly obtained on large areas of several deformed MAX polycrystals; they are also in line with the analysis derived from diffraction data: it is therefore reasonable to extrapolate them to the $3 \mathrm{D}$ behaviour of the polycrystal.

First, the diffraction data and microstructure observations show that the "plastic grains" are characterized by $0^{\circ}<\alpha<70^{\circ}$. In a classical equiaxed microstructure submitted to uniaxial compression, the Schmid law suggests that, on average, the first plastic grains have their slip plane (here the basal plane, supposed to be the main glide system in MAX phases ${ }^{1}$ ) close to $45^{\circ}$ and that the Schmid factor is very small when the slip plane is either parallel or perpendicular to load axis. ${ }^{12}$ Here, the large spanning of $\alpha$ values is associated to deviations from Schmid law and suggests that plasticity is governed by a complex internal stress field, far from the uniaxial case: in particular, the lamellar morphology and the complex interconnection of the grains may lead to a multi-axial stress tensor in the different grain families.

Second, all grain families exhibit a large increase of diffraction peak width: in the "plastic" grains, this can be understood as an increase of both type II and type III microstrains, respectively, related to heterogeneous elastic strains 

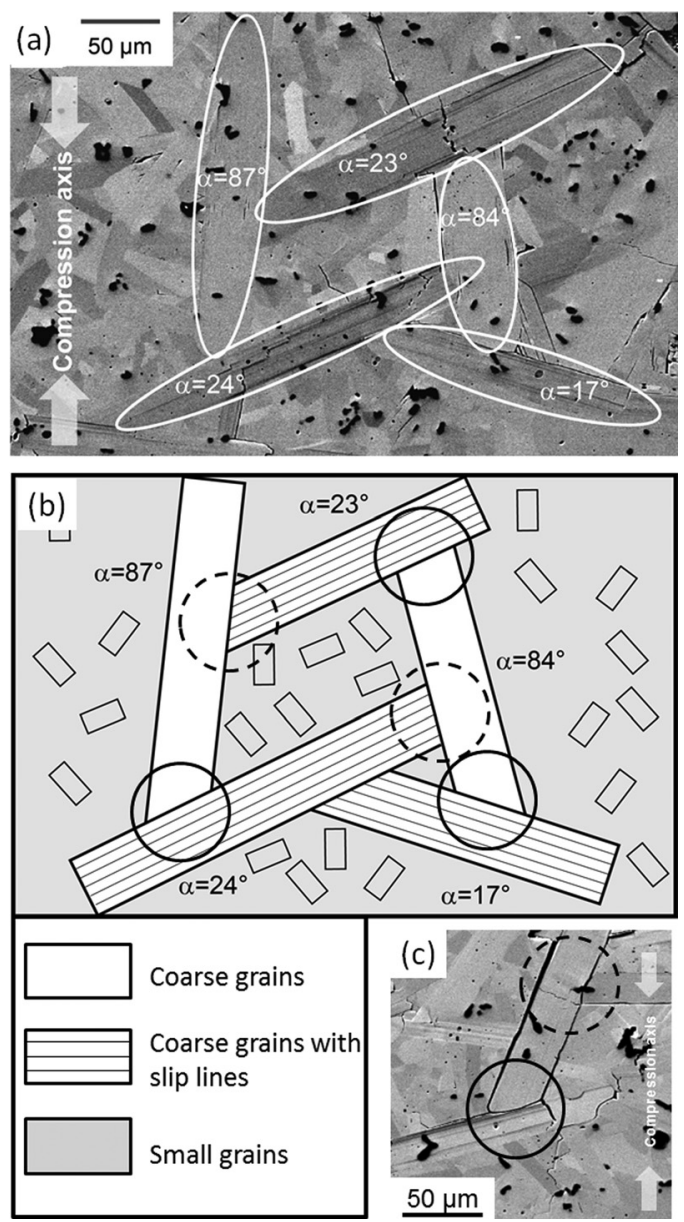

FIG. 5. (a) Post-mortem SEM observation of the in-situ deformed samples. (b) Schematic of the same observed microstructure. (c) Post-mortem SEM micrograph illustrating the grain-to-grain interactions. Solid and dashed circles correspond to the stress concentrations zones.

and dislocations strain field; in the "elastic" grains, this is solely due to an increase of type II heterogeneous elastic strains. In the latter case, a "reversible" mechanism involving dislocation nucleation should be excluded as this would be associated to a reduction of the slope $\left|\partial\left(\varepsilon_{\mathrm{e} 1}\right) / \partial(\sigma)\right|$, i.e., the grain families would belong to "domain 2-plastic" in Fig. 3(a): in other words, they would be considered as "plastic." We must therefore find the origin of the observed large variations of heterogeneous elastic strains in a mechanism involving elastic and plastic grains, with complex arrangement.

It is well established that the mechanical response of a polycrystal depends not only on the absolute value of the macroscopic applied stress but also on its direction, with a reverse (compressive) flow stress often lower than forward (tensile) flow stress. The observed asymmetry-so-called "Bauschinger effect"- of the flow stress is usually ascribed to the build-up of internal stresses due to dislocationmicrostructure interactions and/or directionality of mobile dislocations or annihilation with reverse strain. This asymmetry results in open loops when reversing the macroscopic load. ${ }^{13-15}$ In the case of complex microstructure (such as nanostructured multiphase metals), the presence of very soft and very hard phases leads to the extreme case where open loops are observed even when cycling in tension or compression, i.e., without the need to reverse the load direction. ${ }^{16}$ The open loops are then the footprint of the complex multi-axial stress-strain path undergone by each grain families, as a result of their interactions. These considerations are in agreement with the results of Jones et al. ${ }^{6}$

From this knowledge, and taking into account the specific results reported in this work, we propose the following scenario: ${ }^{17}$

Stage 1: corresponding to "domain 1-elastic" in Figures 3 and 4: when applying a macroscopic compressive load to the "coarse-grain" MAX polycrystal, the lamellar grains close to the parallel to CA (large $\alpha$ ) push on the lamellar grains close to the perpendicular (small $\alpha$ ) to which they are connected (solid circles on Fig. 5(b)), the latter start to bend elastically with a stress concentration at connection point. This is experimentally illustrated in Fig. 5(c). A further increase of the macroscopic load leads to the elastic bending of the large- $\alpha$ lamellar grains that laterally push further on the small- $\alpha$ lamellar grains (dashed circles on Figs. 5(b) and 5(c)).

Stage 2: small- $\alpha$ lamellar grains are subjected to complex multi-axial stresses resulting from bending and stress concentrations: they are the first grains to experience plastic events (dislocation nucleation) and correspond to "domain 2-plastic" in Figures 3 and 4. The other grains (among which are the large- $\alpha$ lamellar grains) remain elastically distorted (as reported in Ref. 6) and correspond to "domain 2-elastic" in Figures 3 and 4.

Stage 3: when removing the load, the internal stress landscape is modified with "plastic" and "elastic" grains retaining, respectively, tensile and compressive residual elastic strains. In the "plastic" grains, only the early plastic regime has been reached: the low density of dislocations nucleated at stress concentration within grain boundaries glide on parallel basal planes and have a high probability to cross the grains without being stored within the grain interior, as suggested by Figure 5(a) with slip traces crossing entirely the "plastic grains:" the dislocations are either stored at opposite grain boundaries or run back to their nucleation site upon unloading.

This scenario agrees well with the diffraction data: stage 1 , associated to macroscopically elastic regime, leads to the build-up of type II heterogeneous elastic strains in all grains and explains the peak broadening observed in the elastic regime (similar behaviour has been recently reported in cubic polycrystalline metals ${ }^{18}$ ). Stage 2 , associated to macroscopically plastic behaviour, results from the combination of plasticity in small- $\alpha$ lamellar grains, exhibiting elastic strain saturation, and of further increase of elastic strain in large- $\alpha$ lamellar grains. Both types of families exhibit a significant peak broadening associated, respectively, to type II + type III microstrains ("plastic grains") and solely type II microstrains ("elastic grains"). Stage 3 explains the apparent recovery of peak broadening, i.e., recovery of microstrains, in the different grain families.

The complex variations of type II and type III microstrains undergone by the different grain families are expected to lead to variations of internal stresses that will result in non-linear elastic strain variations and apparent elastic energy dissipation, even in elastic regime: in this scenario, 
the formation of open stress-strain loops when cycling the MAX polycrystal in the elastic regime can be explained by the complex response of the very anisotropic microstructure. Noteworthy, the same scenario can be applied to "smallgrain" MAX polycrystals as long as they exhibit a lamellar anisotropic microstructure (which is the case here). ${ }^{17}$ The present results add the microstructure anisotropy to the plastic anisotropy as a key ingredient responsible for the very specific mechanical behaviour of MAX polycrystals.

In summary, in situ deformation of $\mathrm{Ti}_{2} \mathrm{AlN}$ polycrystals combined with neutron diffraction and SEM observations allowed for original insights into the MAX phase deformation mechanisms usually explained by the IKB model: the reversible hysteretic loops observed when MAX polycrystals are subjected to elastic load-unload cycles can be as well explained by the complex response of the very anisotropic microstructure that generate strong variations of microstrains in all grains, even in the elastic regime.

This work is based on experiments performed at the Swiss spallation neutron source SINQ, Paul Scherrer Institute, Villigen, Switzerland. This research project has been partially supported by the European Commission under the 7th Framework Programme through the "Research Infrastructures" action of the "Capacities" Programme, NMI3-II Grant No. 283883. This work partially pertains to the French Government program "Investissements
d'Avenir" (LABEX INTERACTIFS, reference ANR-11LABX-0017-01).

${ }^{1}$ M. W. Barsoum, Prog. Solid State Chem. 28, 201 (2000).

${ }^{2}$ Z. Zhang and Z. Sun, Mater. Sci. Eng., A 408, 64 (2005).

${ }^{3}$ M. W. Barsoum, T. Zhen, S. R. Kalidindi, M. Radovic, and A. Murugaiah, Nat. Mater. 2, 107 (2003).

${ }^{4}$ M. W. Barsoum, A. Murugaiah, S. R. Kalidindi, and T. Zhen, Phys. Rev. Lett. 92, 255508 (2004).

${ }^{5}$ M. W. Barsoum, T. Zhen, A. Zhou, S. Basu, and S. R. Kalidindi, Phys. Rev. B 71, 134101 (2005).

${ }^{6}$ N. G. Jones, C. Humphrey, L. D. Connor, O. Wilhelmsson, L. Hultman, H. J. Stone, F. Giuliani, and W. J. Clegg, Acta Mater. 69, 149 (2014).

${ }^{7}$ G. P. Bei, A. Guitton, A. Joulain, V. Gauthier-Brunet, S. Dubois, L. Thilly, and C. Tromas, Philos. Mag. 93, 1784 (2013).

${ }^{8}$ A. Guitton, A. Joulain, L. Thilly, and C. Tromas, Philos. Mag. 92, 4536 (2012).

${ }^{9}$ U. Stuhr, H. Spitzer, J. Egger, A. Hofer, P. Rasmussen, D. Graf, A. Bollhalder, M. Schild, G. Bauer, and W. Wagner, Nucl. Instrum. Methods Phys. Res., Sect. A 545, 330 (2005).

${ }^{10}$ L. Thilly, P. O. Renault, V. Vidal, F. Lecouturier, S. Van Petegem, U. Stuhr, and H. Van Swygenhoven, Appl. Phys. Lett. 88, 191906 (2006).

${ }^{11}$ H. Van Swygenhoven and S. Van Petegem, Mater. Charact. 78, 47 (2013).

${ }^{12}$ J. P. Hirth and J. Lothe, Theory of Dislocations (McGraw-Hill, New York, 1968).

${ }^{13}$ J. Bauschinger, Zivilingenieur 27, 289 (1881).

${ }^{14}$ Y. Xiang and J. J. Vlassak, Acta Mater. 54, 5449 (2006).

${ }^{15}$ Y. Brechet and P. Jarry, J. Phys. III 1, 1985 (1991).

${ }^{16}$ L. Thilly, S. Van Petegem, P. O. Renault, V. Vidal, F. Lecouturier, B. Schmitt, and H. Van Swygenhoven, Appl. Phys. Lett. 90, 241907 (2007).

${ }^{17}$ A. Guitton, Ph.D. thesis, University of Poitiers, 2013.

${ }^{18}$ M. A. Koker, U. Welzel, and E. J. Mittemeijer, J. Appl. Crystallogr. 47, 391 (2014). 